\title{
Fuentes de estrés por aculturación en la infancia y adolescencia. Propuesta del instrumento de medición FEAC-IA*
}

\section{Sources of Acculturation Stress in Childhood and Adolescence. Measuring Instrument Proposal FEAC-IA}

Recepción: 03 Agosto 2019 | Aceptación: 03 Noviembre 2019

\author{
Alfonso Urzúa a \\ Universidad Católica del Norte, Chile \\ ORCID: http://orcid.org/0000-0002-0882-2194 \\ Alejandra Caqueo-Urízar \\ Universidad de Tarapacá, Chile \\ ORCID: http://orcid.org/0000-0002-4614-8380 \\ Jerome Flores \\ Universidad de Tarapacá, Chile \\ ORCID: http://orcid.org/0000-0001-9333-6870
}

a Autor de correspondencia. Correo electrónico: alurzua@ucn.cl

Para citar este artículo: Urzúa, A., Caqueo-Urízar, A., \& Flores, J. (2019). Fuentes de estrés por aculturación en la infancia y adolescencia. Propuesta del instrumento de medición FEAC-IA. Universitas Psychologica, 18(5), 1-10. https://doi.org/10.11144/Javeriana. upsy18-5.fe ai

\section{RESUMEN}

El estrés por aculturación ha sido asociado fuertemente al surgimiento de problemas en la salud de las personas, principalmente en la salud mental. Pese a su importancia como factor de riesgo, su estudio se ha realizado mayoritariamente en población adulta, siendo menor la investigación realizada al respecto en población infantil y adolescente. En esta investigación, se evalúan las propiedades psicométricas del FEACIA, cuestionario destinado a evaluar las fuentes de estrés por aculturación. Se recogieron datos de 401 escolares entre 8 a 18 años $(M=12.4 ; \mathrm{K}$ $=2.5$ ). Se realizaron análisis de confiabilidad y de los indicadores de bondad de ajuste para la evaluación de modelos de la estructura factorial, mediante análisis de ecuaciones estructurales exploratorias (MEEE) y análisis factorial confirmatorio (AFC). El cuestionario presenta buenos indicadores de bondad de ajuste para un modelo trifactorial: añoranza y diferencias con el país de origen, adaptación en la escuela, familia y relación con los pares y experiencias de discriminación $(\mathrm{CFI}=0.98$; TLI $=0.976$; RMSEA $=0.052$ ). La escala propuesta puede constituirse en un aporte importante para recoger insumos en población migrante infantojuvenil sobre las fuentes de estrés, que permitan elaborar planes específicos de intervención.

\section{Palabras clave}

estrés por aculturación; migración; infancia; adolescencia; aculturación.

\begin{abstract}
Acculturation stress has been strongly associated with the emergence of problems in people's health, mainly mental health. Despite its importance as a risk factor, its study has been carried out mainly in the adult population, with less research being carried out on the subject in the child and adolescent population. This research evaluates the psychometric properties of FEAC-IA, a questionnaire designed to evaluate the sources of stress due to acculturation. Data were collected
\end{abstract}


from 401 schoolchildren between 8 and 18 years $(M=12,4$; $\mathrm{SD}=2,5)$. Reliability analysis and fit goodness indicators were performed for the evaluation of factorial structure models through exploratory structural equation analysis (MEEE) and confirmatory factor analysis (AFC). The questionnaire presents good indicators of goodness of fit for a three-factor model: longing and differences with the country of origin, adaptation in school, family and relationship with peers and experiences of discrimination $(\mathrm{CFI}=0.98 ; \mathrm{TLI}=0.976 ; \mathrm{RMSEA}=0.052)$. The proposed scale can be an important contribution to collect inputs in the migrant children and youth population on the sources of stress, which allow the development of specific intervention plans.

Keywords

acculturation stress; migration, childhood; adolescence, acculturation.

La migración puede ser entendida como todo movimiento de personas, independiente del tamaño, composición o causas, hacia el territorio de otro Estado o dentro del mismo, con el fin de residir en este (International Organization for Migration [IOM], 2018). Actualmente, se estima una población cercana a los 258 millones de migrantes internacionales, de los cuales 36.1 millones son niños (Organización Internacional para las Migraciones [OIM], 2018), estimándose que para el año 2050, los migrantes internacionales podrían bordear los 405 millones (OIM, 2010).

Según datos de la Unicef, al año 2018, alrededor de 17 millones de niñas y niños y cinco millones de jóvenes de todo el mundo han sido víctimas de desplazamientos forzosos a causa de la violencia y la guerra, estimando que el número total de niños refugiados y migrantes separados y no acompañados en unos 80 países ha alcanzado un récord histórico, multiplicándose por cinco: de 66000 en 2010 a 300000 en 2016 (Fondo Internacional de Emergencia de las Naciones Unidas para la Infancia [Unicef], 2019). Estos niños y niñas migrarían en busca de sobrevivencia, seguridad, mejoras en su estándar de vida, educación, oportunidades económicas, protección de la explotación y abuso, reunificación familiar o una combinación de estos factores, pudiendo viajar con sus familias, de manera independiente, con un familiar lejano o alguien que no es de su familia (European Forum, 2016)

Como todo movimiento, el migrar implica cambios tanto a nivel de las personas que migran como en el lugar al que llegan. La posibilidad de que estos cambios repercutan en que los menores tengan un proceso sano de adaptación e integración al lugar que llegan, dependerá de la presencia o ausencia de múltiples factores tanto a nivel individual como colectivo, los que por separado o en su interacción pueden facilitar o dificultar este proceso. Existe evidencia de que la presencia de factores de riesgo en el proceso migratorio estaría vinculada a resultados adversos para la salud tanto física como mental de los niños migrantes (Cabieses et al., 2017).

Una de las variables que se ha estudiado en relación con su influencia en el surgimiento de problemas de salud y salud mental es el denominado estrés por aculturación, resultante de este proceso. A nivel psicológico, implica demandas dadas el enfrentamiento de sus actitudes y valores, la necesidad de adquirir nuevas habilidades sociales y normas, los cambios en referencia a la pertenencia a un grupo y el ajuste o adaptación a un ambiente diferente (Berry, 2008). Cuando estas demandas de adaptación a la nueva cultura exceden las capacidades de las personas para afrontarlas, surge el denominado estrés por aculturación (Arbona et al., 2010).

En población adulta, este tipo de estrés se ha asociado a problemas de salud, salud mental y otros (Sternberg \& Lee, 2013; Urzúa, Basabe, Pizarro, \& Ferrer, 2017; Urzúa, CaqueoUrízar, Calderón, \& Rojas, 2017; Urzúa, Ferrer et al., 2017; Urzúa, Heredia, \& CaqueoUrízar, 2016), reportándose incluso la relación entre estrés por aculturación parental con la presencia de síntomas ansiosos en los hijos (Leon, 2014). En adolescentes, su presencia se ha vinculado al desarrollo de sintomatología asociada a problemas de salud mental (Belhadj \& Koglin, 2015; Belhadj, Koglin, \& Petermann, 2014). Pese a su importancia, especialmente con fines preventivos, la gran mayoría de los estudios en estrés por aculturación se ha realizado en población adulta, encontrándose 
solo dos instrumentos que evalúan esta variable en población infantil y adolescente: el SAFE (y su versión abreviada SAFE-C) y el ASIC.

La escala SAFE (Societal, Attitudinal, Familial, and Environmental Acculturative Stress) es un instrumento de 60 ítems propuesto para adolescentes por Padilla y colaboradores (Mena, Padilla, \& Maldonado, 1987; Padilla, Álvarez, \& Lindholm, 1986), la cual presenta una versión abreviada de 17 ítems (Mena et al., 1987), y mide el estrés aculturativo en estudiantes universitarios en las dimensiones social, actitudinal, familiar y ambiental. Esta escala fue modificada luego para niños entre $8 \mathrm{y}$ 12 años por Chávez, Moran, Reid y López (1997).

La escala ASIC (Acculturative Stress Inventory for Children) fue propuesta por Suárez-Morales, Dillon y Szapocznik (2007), intentando resolver los problemas metodológicos presentes en el desarrollo de la escala SAFE. Estos autores proponen una escala de 12 reactivos, agrupados en dos factores independientes: discriminación percibida y sentimientos de ser excluidos o marginalizados.

Dado que esta última escala entrega información acerca de niveles de discriminación y marginación percibidos, pero no de las posibles fuentes que las originan, es que, con miras a aportar insumos para el diseño de intervenciones en población infantil y adolescente, se construye una escala que permite identificar posibles fuentes de estrés. De la literatura revisada, no ha sido posible encontrar modelos teóricos o evidencia empírica que aporte información acerca de las fuentes de estrés en niños y adolescentes migrantes. En este marco, el objetivo de este estudio fue valorar las propiedades psicométricas de una escala destinada a evaluar las fuentes de estrés por aculturación, en menores migrantes de 8 a 18 años.

\section{Método}

Se realiza un estudio de tipo instrumental, mediante el uso de ecuaciones estructurales exploratorias, dado que es menos restrictivo que un análisis factorial confirmatorio (Marsh, Morin, Parker, \& Kaur, 2014), toda vez que se acerca más a la forma en que las variables se presentan en la realidad, sin cargas exclusivas en un factor determinado (Howard, Gagné, \& Forest, 2018).

\section{Participantes}

La muestra inicial incluyó 401 escolares de ambos sexos entre cuarto año de enseñanza básica (primaria) hasta el último año de enseñanza secundaria, provenientes de diferentes establecimientos públicos, subvencionados y privados de la ciudad de Arica (Chile). Se consideró como criterio de inclusión un rango de edad entre 8 a 18 años, y no haber nacido en Chile.

Del total de cuestionarios recolectados, se descartaron 22 por identificarse como nacidos en Chile y 34 por presentar varianza cero en sus respuestas a lo largo del cuestionario, puesto que no aportan información útil al análisis (Hefetz \& Liberman, 2017). No se registró varianza cero a lo largo de los ítems. Un $5 \%$ de los instrumentos tenía algunos ítems en blanco y estos no eran superiores a un $25 \%$ del total de ítems. Los 8 cuestionarios que tenían más de ese porcentaje en blanco no fueron incluidos. La muestra final se conformó por 337 estudiantes entre 8 y 18 años, con una media de 12.4 y una desviación estándar de 2.5 años. Un $47.8 \%$ son mujeres y un 52.2 $\%$ son hombres. Al desagregar por país de origen, la gran mayoría son nacidos en Bolivia (47.2\%) y Perú (32.3\%), seguidos de Colombia (6.2\%), Venezuela (5.3\%), Ecuador (1.8\%), Argentina (0.9\%). Un $6.2 \%$ correspondió a otros países.

\section{Procedimientos}

Este estudio es parte de un proyecto mayor del Centro de Justicia Educacional y contó con la aprobación del comité de ética de la Universidad de Tarapacá. Con base en una revisión teórica sobre estrés en niños migrantes y a la experiencia de investigación en niños y adolescentes migrantes, realizados 
en la ciudad de Antofagasta (Caqueo-Urízar et al., 2017), se construye un instrumento compuesto por 17 reactivos que inquieren sobre tres fuentes distintas de estrés por aculturación. Posteriormente al consentimiento de padres y asentimiento a los estudiantes, se realiza un muestreo piloto y entrevistas cognitivas a 15 estudiantes en la ciudad de Antofagasta, para verificar la adecuada comprensión de los ítems. Las entrevistas realizadas, destinadas a medir la comprensión de cada una de las preguntas del cuestionario (en tanto forma y contenido), permitieron mantener la estructura original de los reactivos sin realizar ningún cambio.

Una vez comprobada la adecuada comprensión del instrumento, se invitó a participar del estudio a 42 establecimientos educacionales de la ciudad de Arica. Un $69 \%$ accedió a participar del estudio, considerándose 29 establecimientos en total. En estos establecimientos, se solicitaron los consentimientos a los padres y posteriormente los asentimientos a los mismos estudiantes. Cada aplicación duró aproximadamente 45 minutos. El tiempo medio en responder fue de 10 minutos. $\mathrm{Al}$ menos dos encuestadores estuvieron presentes por sala para responder las dudas, aparte del profesor del mismo establecimiento.

\section{Instrumento}

El instrumento inicial consideró 17 ítems de respuesta tipo Likert de seis opciones. La consigna fue: "Evalúa si las siguientes situaciones te han sucedido en los últimos seis meses y cuánto te han afectado". Las opciones de respuesta van desde cero (No he tenido este problema) a 6 (Me ha afectado mucho).

Las dimensiones propuestas para evaluar las fuentes de estrés fueron:

1. Añoranza y diferencias con el país de origen: compuesta por cuatro ítems centrados en establecer en qué medida los estudiantes extrañan su país de origen ("Extraño a los amigos que tenía en mi país") y lo encuentran diferente del país en que actualmente residen ("Creo que este país es muy diferente al mío").
2. Adaptación en la escuela, familia y relación con los pares: consideran siete reactivos los que se centran en la adaptación que los niños, niñas y adolescentes tienen a su escuela ("Me cuesta adaptarme a la escuela [colegio]"), familia ("Mi familia se pelea más ahora que antes [en mi país]") y con sus pares ("No me llevo bien con los niños de este país").

3. Experiencias de discriminación: comprende seis reactivos que abordan diferentes experiencias en que los participantes puedan sentirse discriminados ("A veces me miran mal por ser extranjero").

\section{Análisis de datos}

Dado que el análisis de ecuaciones estructurales exploratorias (Modelo de Ecuaciones Estructurales Exploratorias [MEEE]) puede ser considerado más apropiado para analizar la estructura de los instrumentos puesto que es menos restrictivo que análisis factorial confirmatorio (Marsh, Nagengast, \& Morin, 2013), y a que se acerca más a la forma en que las variables se presentan en la realidad, sin cargas exclusivas en un factor determinado (Howard et al., 2018), se decide realizar de manera paralela un análisis factorial confirmatorio (AFC) y un MEEE. Marsh et al. (2014) recomiendan comparar simultáneamente los resultados obtenidos a partir de MEEE y AFC, con el fin de tener una perspectiva más completa, considerando que si ambos no difieren se puede retener el modelo AFC. Adicionalmente, Booth y Hughes (2014) coinciden con la utilidad de comparar ambos análisis, si bien destacan que el AFC ofrece una mayor posibilidad de interpretación.

Se utilizó el programa SPSS para obtener los valores descriptivos y realizar comparaciones entre grupos y el programa MPLUS para realizar análisis de ecuaciones estructurales exploratorias (MEEE) y análisis factorial confirmatorio (AFC) (Asparouhov \& Muthén, 2009; Muthén \& Muthén, 2017). Se optó por una rotación oblicua dado que es lo recomendado en ciencias sociales (Lloret-Segura, Ferreres-Traver, Hernández-Baeza, \& Tomás-Marco, 2014). La 
rotación seleccionada fue quartimin. El análisis se realizó en cinco fases: estadísticos descriptivos, comparación de grupos por sexo, etnia y edad, análisis factorial del instrumento con tres y cuatro factores, propuesta de un instrumento breve y, finalmente, análisis de la fiabilidad del instrumento y sus escalas.

Para los indicadores de bondad de ajuste del modelo, se consideró un RMSA $<0.08$ como aceptable y $<0.05$ como bueno, mientras que un CFI y TLI $>0.9$ como adecuado y $>0.95$ como óptimo (Brown, 2015; Hu \& Bentler, 1999; McDonald \& Ho, 2002). El chi cuadrado no fue considerado, dado los problemas que presenta con muestras mayores a 200 y en casos de distribución no normal, como es el presente caso. El ratio $\chi 2 / \mathrm{df}$ se considera adecuado $<3$ (Brown, 2015; Hu \& Bentler, 1999). La fiabilidad de las escalas se consideró adecuada a partir de 0.7 de acuerdo con Nunnally (1987) y Morales (2007). Se ha tenido en consideración, en el presente estudio, la propuesta de Fornell y Larcker (1981) sobre considerar la fiabilidad compuesta y varianza extraída, para una mejor interpretación. El criterio mínimo que se esperaría es que la primera alcanzase 0.7 y la segunda 0.5 , en cada factor.

Dado que se generó un efecto de suelo (floor efect) en las respuestas al tender más de un $15 \%$ a una sola opción, se consideraron las variables como categóricas (Bot et al., 2003). Se utilizó el método estimación de mínimos cuadrados ponderados robustos (WLSMV) que es más adecuado cuando los datos no se distribuyen normalmente (Lloret-Segura et al., 2014; Li, 2016; Muthén \& Muthén, 2017).

\section{Resultados}

\section{Participantes}

Entre los 401 instrumentos recolectados, se descartaron 22 por identificarse como nacidos en Chile, y 34 por presentar varianza cero en sus respuestas a lo largo del cuestionario, puesto que no aportan información útil al análisis (Hefetz \& Liberman, 2017). No se registró varianza cero a lo largo de los ítems. Un $5 \%$ de los instrumentos tenía algunos ítems en blanco y estos no eran superiores a un $25 \%$ del total. Los ocho instrumentos que tenían más de ese porcentaje en blanco no se incluyeron.

La muestra final se conformó de 337 estudiantes entre 8 y 19 años, con una media de 12.4 y una desviación estándar de 2.5. Un $47.8 \%$ eran mujeres y un $52.2 \%$, hombres. Al desagregar por país de origen, un $32.3 \%$ eran peruanos, un $47.2 \%$, bolivianos y el resto se distribuye en otras nacionalidades. Respecto a su etnia, un $45 \%$ se declara aymara, un $6.8 \%$, quechua, un $1.2 \%$, mapuche y un $1.5 \%$, afrodescendiente.

\section{Descriptivos}

El promedio del puntaje total entre los participantes fue de 19.5 con una desviación estándar de 15 puntos. El 50 \% de ellos estaba bajo los 17 puntos en la escala (Tabla 1). Se incluye el porcentaje de respuestas cero, puesto que es preciso mostrar el efecto piso cuando este se presente (Bot et al., 2003), aun cuando al considerar los datos como categóricos y con el estimador WLMSV se puede manejar adecuadamente este efecto (Brown, 2015). Puede observarse que al menos tres ítems van más allá de los limites tradicionalmente aceptados de \pm 2 para la asimetría y la curtosis (George \& Mallery, 2010). Se calcularon pruebas t para muestras independientes, con el propósito de comparar grupos por edad (primaria/ secundaria) y sexo, no encontrándose diferencias significativas en ninguna de ellas. 
Tabla 1

Estadísticos descriptivos reactivos escala y edad

\begin{tabular}{|c|c|c|c|c|c|c|c|}
\hline \multirow[b]{2}{*}{ Ítems } & \multirow{2}{*}{$\begin{array}{c}\text { Media } \\
\text { Estadistico }\end{array}$} & \multirow{2}{*}{$\begin{array}{c}D E \\
\text { Estadístico }\end{array}$} & \multicolumn{2}{|c|}{ Asimetría } & \multicolumn{2}{|c|}{ Curtosis } & \multirow{2}{*}{$\begin{array}{c}\% \\
\text { Efecto } \\
\text { piso }\end{array}$} \\
\hline & & & Estadístico & $\begin{array}{c}\text { Error } \\
\text { estándar }\end{array}$ & Estadístico & $\begin{array}{l}\text { Error } \\
\text { estándar }\end{array}$ & \\
\hline Edad & 12.39 & 2.53 & 0.447 & 0.123 & -0.754 & 0.246 & \\
\hline FEACI & 2.504 & 1.8567 & 0.05 & 133 & 1.377 & 0.265 & 21.4 \\
\hline $\mathrm{EAC} 2$ & 561 & .8003 & .01 & 133 & 1.328 & 0.265 & 18.4 \\
\hline $\mathrm{AC} 3$ & 1.181 & 5814 & 1.228 & 133 & 156 & 0.265 & 57 \\
\hline $\mathrm{F}$ & & 5367 & & 3 & 21 & 265 & 57 \\
\hline EAC & 2 & 6782 & 1.035 & 133 & -0.196 & .265 & 51.6 \\
\hline$A$ & 1 & 7107 & 1.26 & 133 & 0.162 & 0.265 & 57.3 \\
\hline $\mathrm{F}$ & & & & & & 265 & 74.8 \\
\hline & & & & & & 65 & 64.7 \\
\hline EAC9 & 1 & 6588 & 1.358 & .133 & 0.493 & 0.265 & 59.6 \\
\hline $\mathrm{EAC10}$ & & 1014 & 354 & 133 & -1.583 & 0.265 & 40.1 \\
\hline $\mathrm{AC} 11$ & & & & 133 & 2.576 & 0.265 & 68 \\
\hline & & & & & & 65 & 57.9 \\
\hline $\mathrm{F}$ & & & 1. & 33 & 829 & .265 & 69.4 \\
\hline $\mathrm{ACl} 14$ & & 3 & 1.66 & 133 & 377 & 0.265 & 68.8 \\
\hline FEAC15 & 0.561 & 1.2548 & 467 & 0.133 & 5.285 & 0.265 & 76.9 \\
\hline & & & & & .786 & .265 & 87.2 \\
\hline FEAC17 & 0.409 & 1.0931 & 3.039 & 0.133 & 8.655 & 0.265 & 82.5 \\
\hline
\end{tabular}

\section{Análisis factorial}

Se comparan los resultados de los indicadores de bondad de ajuste obtenidos a partir del MEEE para los modelos de 3 y 4 factores (Tabla 2). Un ítem fue eliminado de entrada, puesto que generó una matriz negativa, lo que de acuerdo con el programa SPSS puede deberse a una dependencia linear de este ítem con un factor.

\section{Tabla 2}

Indicadores de bondad de ajuste para modelos de tres y cuatro factores

\begin{tabular}{|c|c|c|c|c|c|c|c|c|}
\hline Factores & $x^{2}$ & $d f$ & $\begin{array}{c}\chi^{2 /} / \\
d f\end{array}$ & $p$ & CFI & TLI & RMSEA & $\begin{array}{l}\text { RMSEA } \\
90 \% \text { IC }\end{array}$ \\
\hline MEEE 3 Factores & 156.243 & 75 & 2.1 & 0 & 0.977 & 0.964 & 0.057 & $0.044-0.069$ \\
\hline CFA 3 Factores & 269.36 & 101 & 2.7 & 0 & 0.953 & 0.945 & 0.07 & $0.06-0.081$ \\
\hline MEEE 4 Factores & 109.693 & 62 & 1.8 & 0.0002 & 0.987 & 0.974 & 0.048 & $0.033-0.062$ \\
\hline CFA 4 Factores & 255.294 & 98 & 2.6 & 0 & 0.956 & 0.947 & 0.069 & $0.059-0.079$ \\
\hline
\end{tabular}

Las Tablas 3 y 4 muestran las cargas factoriales para los modelos de tres y cuatro factores tanto desde el análisis mediante MEEE como desde el AFC.
Tabla 3

Modelo de tres factores inicial

\begin{tabular}{lrrrrrr}
\hline \multirow{2}{*}{ Ítem } & \multicolumn{3}{c}{ MEEE } & \multicolumn{3}{c}{ CFA } \\
\cline { 2 - 7 } & \multicolumn{1}{c}{$F 1$} & \multicolumn{1}{c}{$F 2$} & \multicolumn{1}{c}{$F 3$} & $F 1$ & $F 2$ & $F 3$ \\
\hline FEAC1 & $\mathbf{0 . 7 8}$ & -0.008 & -0.021 & $\mathbf{0 . 6 5 8}$ & & \\
FEAC2 & $\mathbf{0 . 7 6 2}$ & -0.009 & -0.02 & $\mathbf{0 . 6 3 6}$ & & \\
FEAC3 & 0.119 & $\mathbf{0 . 5 1 4}$ & 0.151 & & & $\mathbf{0 . 6 9 1}$ \\
FEAC4 & 0.207 & 0.164 & $\mathbf{0 . 5 1 2}$ & & & $\mathbf{0 . 7 3 8}$ \\
FEAC5 & 0.235 & 0.284 & 0.285 & & & $\mathbf{0 . 6 5 8}$ \\
FEAC6 & 0.118 & $\mathbf{0 . 8 2 9}$ & -0.116 & $\mathbf{0 . 7 7}$ & \\
FEAC7 & -0.094 & $\mathbf{0 . 3 7 7}$ & $\mathbf{0 . 3 4 2}$ & $\mathbf{0 . 6 3 7}$ & \\
FEAC8 & 0.052 & 0.246 & $\mathbf{0 . 5 8 6}$ & & & $\mathbf{0 . 8 0 6}$ \\
FEAC9 & 0.188 & 0.149 & 0.293 & $\mathbf{0 . 6 5 2}$ & & \\
FEAC10 & $\mathbf{0 . 6 1 1}$ & 0.043 & 0.18 & $\mathbf{0 . 7 7 8}$ & & \\
FEAC11 & 0.024 & $\mathbf{0 . 8 8 2}$ & -0.012 & & $\mathbf{0 . 8 7 4}$ & \\
FEAC12 & 0.036 & -0.071 & $\mathbf{0 . 8 8 3}$ & & $\mathbf{0 . 7 5 8}$ \\
FEAC13 & 0.01 & 0.192 & $\mathbf{0 . 4 1 2}$ & & $\mathbf{0 . 5 6 8}$ \\
FEAC14 & -0.121 & 0.12 & $\mathbf{0 . 5 4 9}$ & & $\mathbf{0 . 5 5 5}$ \\
FEAC15 & -0.094 & $\mathbf{0 . 8 5 3}$ & 0.07 & $\mathbf{0 . 8 6 3}$ & \\
FEAC17 & -0.052 & $\mathbf{0 . 6 9 1}$ & 0.145 & $\mathbf{0 . 7 9 3}$ & \\
\hline
\end{tabular}

Nota.En negrita se destacan las cargas factoriales mayores a 0.3. MEEE: Modelo ecuaciones estructurales exploratorias; CFA: Análisis factorial confirmatorio

Tabla 4

Modelo de cuatro factores inicial

\begin{tabular}{rrrrrrrrr}
\hline \multirow{2}{*}{ Ítem } & \multicolumn{9}{c}{$M E E E$} & \multicolumn{1}{c}{ CFA } \\
\cline { 2 - 10 } & \multicolumn{1}{c}{$F 1$} & \multicolumn{1}{c}{$F 2$} & \multicolumn{1}{c}{$F 3$} & \multicolumn{1}{c}{$F 4$} & \multicolumn{1}{c}{$F 1$} & $F 2$ & $F 3$ & $F 4$ \\
\hline FEAC1 & $\mathbf{0 . 8 3 5}$ & 0.03 & -0.124 & -0.018 & $\mathbf{0 . 6 5 8}$ & & & \\
FEAC2 & $\mathbf{0 . 7 1 6}$ & -0.056 & 0.238 & -0.03 & $\mathbf{0 . 6 3 9}$ & & & \\
FEAC3 & 0.021 & $\mathbf{0 . 5 0 7}$ & $\mathbf{0 . 4 0 1}$ & 0.046 & & & $\mathbf{0 . 7 1}$ & \\
FEAC4 & 0.107 & 0.076 & $\mathbf{0 . 4 0 5}$ & $\mathbf{0 . 5 0 2}$ & & & $\mathbf{0 . 7 6}$ & \\
FEAC5 & 0.167 & 0.237 & $\mathbf{0 . 3 0 2}$ & 0.251 & & & $\mathbf{0 . 6 7 6}$ & \\
FEAC6 & 0.103 & $\mathbf{0 . 7 9 5}$ & 0.093 & -0.109 & & $\mathbf{0 . 7 7}$ & & \\
FEAC7 & -0.126 & $\mathbf{0 . 3 9 6}$ & 0.171 & 0.274 & & $\mathbf{0 . 6 3 7}$ & & $\mathbf{0 . 8 4 8}$ \\
FEAC8 & 0.006 & 0.249 & 0.209 & $\mathbf{0 . 5 3 5}$ & & & & \\
FEAC9 & 0.175 & 0.191 & 0.083 & 0.234 & $\mathbf{0 . 6 5 1}$ & & & \\
FEAC10 & $\mathbf{0 . 6 2 8}$ & 0.082 & -0.081 & 0.191 & $\mathbf{0 . 7 7 6}$ & & & \\
FEAC11 & 0.04 & $\mathbf{0 . 8 6 8}$ & 0.013 & -0.006 & & $\mathbf{0 . 8 7 4}$ & & $\mathbf{0 . 7 9 4}$ \\
FEAC12 & 0.058 & -0.034 & -0.019 & $\mathbf{0 . 8 7 8}$ & & & & $\mathbf{0 . 5 9 5}$ \\
FEAC13 & 0.075 & 0.269 & -0.184 & $\mathbf{0 . 3 9 3}$ & & & & $\mathbf{0 . 5 8 1}$ \\
FEAC14 & -0.055 & 0.204 & -0.195 & $\mathbf{0 . 5 2 7}$ & & & \\
FEAC15 & -0.042 & $\mathbf{0 . 8 8 1}$ & -0.104 & 0.063 & & $\mathbf{0 . 8 6 3}$ & & \\
FEAC17 & -0.017 & $\mathbf{0 . 7 1 2}$ & -0.034 & 0.127 & & $\mathbf{0 . 7 9 3}$ & \\
\hline
\end{tabular}

Nota.En negrita se destacan las cargas factoriales mayores a 0.3. MEEE:

ecuaciones estructurales exploratorias; CFA: Análisis factorial confirmatorio

Propuesta de un instrumento breve

Con el fin de obtener una versión más breve y con mejores indicadores de bondad de ajuste, se eliminaron los ítems 5 y 9 , los que poseen cargas en el límite del 0.3 y el 3 , con el fin de definir mejor la estructura factorial. Por ende, la versión breve consta de 13 ítems, cuya carga factorial 
analizada a través de MEEE y CFA puede ser observada en la Tabla 5.

\section{Tabla 5}

Modelo final de tres factores

\begin{tabular}{|c|c|c|c|c|c|c|}
\hline \multirow{2}{*}{ Ítem } & \multicolumn{3}{|c|}{ MEEE } & \multicolumn{3}{|c|}{ CFA } \\
\hline & $F I$ & $F 2$ & $F^{3}$ & $F 1$ & $F 2$ & $F_{3}$ \\
\hline FEACl Extraño (echo de menos) mi país de origen & 0.8 & 0.042 & -0.049 & 0.722 & & \\
\hline FEAC2 Creo que este pais es muy diferente al mío & 0.732 & -0.074 & 0.074 & 0.679 & & \\
\hline FEAC4 Me cuesta adaptarme a la escuela (colegio) & 0.168 & 0.081 & 0.586 & & & 0.726 \\
\hline $\begin{array}{l}\text { FEAC6 Los otros niños me hacen sentir como un } \\
\text { extranjero siempre }\end{array}$ & 0.119 & 0.804 & -0.091 & & 0.761 & \\
\hline $\begin{array}{l}\text { FEAC7 Creo que los profesores no me tratan igual } \\
\text { que al resto }\end{array}$ & -0.126 & 0.388 & 0.308 & & 0.613 & \\
\hline FEAC8 Me cuesta juntarme con niños chilenos & 0.035 & 0.181 & 0.655 & & & 0.829 \\
\hline FEAC10 Extraño a los amigos que tenía en mi país & 0.599 & 0.112 & 0.137 & 0.833 & & \\
\hline FEAC11 A veces me miran mal por ser extranjero & 0.047 & 0.865 & 0.003 & & 0.878 & \\
\hline FEAC12 Me cuesta mucho hacer amigos nuevos & 0.028 & -0.072 & 0.89 & & & 0.789 \\
\hline $\begin{array}{l}\text { FEAC13 Mi familia se pelea más ahora que antes } \\
\text { (en mi país) }\end{array}$ & 0.023 & 0.236 & 0.381 & & & 0.609 \\
\hline $\begin{array}{l}\text { FEAC1 } 4 \text { Me cuesta juntarme con niños de mi } \\
\text { propio pais }\end{array}$ & -0.115 & 0.156 & 0.513 & & & 0.587 \\
\hline $\begin{array}{l}\text { FEAC15 En la escuela mis compañeros me } \\
\text { molestan por ser extranjero }\end{array}$ & -0.06 & 0.851 & 0.068 & & 0.88 & \\
\hline $\begin{array}{l}\text { FEAC17 Me han agredido fisicamente por venir de } \\
\text { otro país }\end{array}$ & -0.054 & 0.734 & 0.092 & & 0.789 & \\
\hline
\end{tabular}

Nota.En negrita se destacan las cargas factoriales mayores a 0.3. MEEE: ecuaciones estructurales exploratorias; CFA: Análisis factorial confirmatorio $F 1=$ Factor Añoranza y diferencias con el país de origen: ítems 1,2 y $10 . F 2=$ Factor Adaptación en la Escuela, familia y relación con los pares: ítems 4 , 8, 12, 13 y 14. F3=Factor Experiencias de discriminación: ítems 6, 7, 11, 15 y 17.

Los indicadores de bondad de ajuste de esta solución factorial puede ser observada en la Tabla 6.

\section{Tabla 6}

Indicadores de bondad de ajuste para modelo final de trece reactivos y tres factores

\begin{tabular}{|c|c|c|c|c|c|c|c|c|}
\hline Modelo final & $x^{2}$ & $d f$ & $\begin{array}{l}x^{2 /} / \\
d f\end{array}$ & $p$ & CFI & TLI & RMSEA & $\begin{array}{l}\text { RMSEA 90\% } \\
\text { IC }\end{array}$ \\
\hline MEEE 3 factores & 72.321 & 42 & 1.7 & 0.002 & 0.99 & 0.981 & 0.046 & $0.027-0.064$ \\
\hline CFA 3 factores & 118.345 & 62 & 1.9 & 0 & 0.981 & 0.976 & 0.052 & $0.038-0.066$ \\
\hline
\end{tabular}

Nota 2 2: Chi cuadrado. $d f$ : grados de

libertad. $\chi^{2}$ / $d f$ : ratio chi cuadrado

La fiabilidad de las subescalas en la versión final de tres factores es: Añoranza y diferencias con el país de origen 0.728 , adaptación en la escuela, familia y relación con los pares 0.738 y experiencias de discriminación 0.767 . Puede apreciarse en la Tabla 7 que la fiabilidad compuesta es superior al $\alpha$ y que la varianza extraída es mayor al $40 \%$ necesario.
Tabla 7

Fiabilidad tradicional, compuesta y varianza extraída

\begin{tabular}{lrrr}
\hline Indicador & Factor 1 & Factor 2 & \multicolumn{1}{l}{ Factor 3 } \\
\hline $\mathrm{N}$ & 3 & 3 & 5 \\
$\mathrm{VE}$ & 0.56 & 0.62 & 0.51 \\
$\mathrm{FC}$ & 0.79 & 0.89 & 0.84 \\
$\alpha$ & 0.73 & 0.74 & 0.77 \\
\hline
\end{tabular}

Nota.N = número de ítems por factor,

$\mathrm{VE}=$ Varianza extraída, $\mathrm{FC}=$ Fiabilidad compuesta. A = Alpha de Cronbach

\section{Discusión}

En el instrumento original, se opta por mantener la estructura de tres factores tanto por su parsimonia como por ser más estable al considerar más ítems por factor. El ítem 16 fue descartado del análisis y es posible que tanto su curtosis como asimetrías extremas generasen una dependencia linear con uno de los factores. Es posible que este reactivo haya sido redactado en una forma menos común de la situación de discriminación desde los profesores hacia los estudiantes. El ítem 7 va en la misma línea, pero al ser una experiencia más frecuente posiblemente evitó dificultades en los análisis. En todo caso, este problema no hubiese sido posible detectarlo a partir de un tradicional AFE, por lo que la utilización del MEEE ha aportado ventaja.

En su forma original, el instrumento FEAC cuenta con indicadores de bondad de ajuste adecuados, y su estructura factorial propuesta originalmente cuenta con suficiente respaldo en los datos dada la distribución y cargas factoriales. La versión breve mejora los indicadores de bondad de ajuste llegando al nivel óptimo, adicionalmente, acercando al máximo los modelos MEEE y CFA.

Las dimensiones propuestas originalmente reciben respaldo de los datos, siendo coherentes tanto en MEEE como en CFA. La cantidad de ítems por factor es igual o mayor a tres, lo que le permite mayor estabilidad a la dimensión (LloretSegura et al., 2014).

En relación con la estructura teórica de los factores, lamentablemente no fue posible 
encontrar modelos teóricos que pudieran ser contrastados, por lo que esto se convierte en una posible limitante del estudio, a la vez que una fortaleza, ya que significa el comienzo de una línea de estudios en esta área. Adicionalmente, resulta necesario profundizar luego los estudios de esta escala en otras poblaciones de migrantes (haitianos, por ejemplo) y analizar evidencias de validez tanto convergente como discriminante, comparando sus resultados con variables asociadas (por ejemplo, sintomatología de salud mental, bienestar, calidad de vida).

\section{Conclusiones}

El FEAC-IA pareciera ser un buen instrumento destinado a evaluar la presencia de estrés por aculturación en los tres factores señalados, pudiendo constituirse en una herramienta adecuada para su uso en población migrante, con miras a obtener insumos para la elaboración de planes de intervención o pesquisar factores de riesgo para salud.

\section{Referencias}

Arbona, C., Olvera, N., Rodríguez, N., Hagan, J., Linares, A., \& Wiesner, M. (2010). Acculturative stress among documented and undocumented Latino immigrants in the United States. Hispanic Journal of Behavioral Sciences, 32(3), 362-384. https ://doi.org/10.1177/0739986310373210

Asparouhov, T., \& Muthén, B. (2009). Exploratory structural equation modeling. Structural Equation Modeling, 16, 397-438. https://doi.org/10.1080/10705510 903008204

Belhadj, E., \& Koglin, U. (2015). Emotional and behavioral problems in migrant children and adolescents in American countries: A systematic review. Journal of Immigrant Minority Health, 17(4), 1240-1258. https:// doi.org/10.1007/s10903-014-0039-2

Belhadj, E., Koglin, U., \& Petermann, F. (2014). Emotional and behavioral problems in migrant children and adolescents in
Europe: A systematic review. European Child Eु Adolescent Psychiatry, 23(6), 373-391. https://doi.org/10.1007/s00787-0 13-0485-8.

Berry, J. W. (2008). Globalization and acculturation. International Journal of Intercultural Relations, 32(4), 328-336. http s://doi.org/10.1016/j.ijintrel.2008.04.001

Booth, T., \& Hughes, D. J. (2014). Exploratory structural equation modeling of personality data. Assessment, 21(3), 260-271. https://d oi.org/10.1177/1073191114528029

Bot, S. D. M., Terwee, C. B., van der Windt, D. A. W. M., Bouter, L. M., Dekker, J. M., \& de Vet, H. C. W. (2003). Psychometric evaluation of self-report questionnaires: The development of a checklist. En H. J. Adér \& C. Mellenberg (Eds.), Proceedings of the second workshop on research methodology (pp. 161-168). Amsterdam: VU University.

Brown, T. A. (2015). Methodology in the social sciences. Confirmatory factor analysis for applied research (2.a ed.). Nueva York: Guilford Press.

Cabieses, B., Chepo, M., Oyarte, M., Markkula, N., Bustos, P., Pedrero, V., \& Delgado, I. (2017), Brechas de desigualdad en salud en niños migrantes versus locales en Chile. Revista Chilena de Pediatría, 88(6), 707-716. Recuperado de http://www.revistachilenadepediatria.cl/ index.php/rchped/article/view/71

Caqueo-Urízar, A., Urzúa, A., Alquinta, B., Jeria, R., Jorquera, R., \& Lau, X. (2017). Overall life satisfaction of Latin American immigrant children in northern Chile. International Journal of Management and Applied Science, 3(3), 48-49. Recuperado de http://www.iraj.in/journal/journal_file/j ournal_pdf/14-349-149691397748-49.pdf

Chávez, D. V., Moran, V. R., Reid, S. L., \& López, M. (1997). Acculturative stress in children: A modification of the SAFE scale. Hispanic Journal of Behavioral Sciences, 19(1), 34-44. https://doi.org/10.1177/0739 9863970191002

European Forum. (2016). Report of 10th European Forum on the rights of the child: 
The protection of children in migration. Recuperado de http://ec.europa.eu/newsro om/document.cfm?doc_id $=42359$

Fornell, C., \& Larcker, D. F. (1981). Evaluating structural equation models with unobservable variables and measurement error. Journal of Marketing Research, 18(1), 39-50. https://doi.org/10.1177/0022243781 01800104

George, D., \& Mallery, M. (2010). SPSS for windows step by step: A simple guide and reference, 17.0 update (10.a ed.). Boston: Pearson.

Hefetz, A., \& Liberman, G. (2017). The factor analysis procedure for exploration: A short guide with examples. Cultura y Educación, 29(3), 395-408. https://doi.org/10.1080/11 356405.2017.1365425

Howard, J. L., Gagné, M., S., A. J., \& Forest, J. (2018). Using bifactor exploratory structural equation modeling to test for a continuum structure of motivation. Journal of Management, 44(7), 2638-2664. https://d oi.org/10.1177/0149206316645653

Hu, L. -T., \& Bentler, P. M. (1999). Cutoff criteria for fit indexes in covariance structure analysis: Conventional criteria versus new alternatives. Structural Equation Modeling: A Multidisciplinary Journal, 6(1), 1-55. http s://doi.org/10.1080/10705519909540118

International Organization for Migration. (2018). Informe sobre las migraciones en el mundo. Ginebra: Autor.

Leon, A. L. (2014). Immigration and stress: the relationship between parents' acculturative stress and young children's anxiety symptoms. Inquiries Journal/Student Pulse, 6(3), 1-19. Recuperado de http://ww w.inquiriesjournal.com/a?id $=861$

Li, C. H. (2016). Confirmatory factor analysis with ordinal data: Comparing robust maximum likelihood and diagonally weighted least squares. Behavior Research Methods, 48, 936-949. https://doi.org/10.37 58/s13428-015-0619-7

Lloret-Segura, S., Ferreres-Traver, A., Hernández-Baeza, A., \& Tomás-Marco, I. (2014). El análisis factorial exploratorio de los ítems: una guía práctica, revisada y actualizada. Anales de Psicología, 30(3), 1151-1169. Recuperado de https://www.re dalyc.org/pdf/167/16731690031.pdf

Marsh, H. W., Nagengast, B., \& Morin, A. J. S. (2013). Measurement invariance of bigfive factors over the life span: MEEE tests of gender, age, plasticity, maturity, and la dolce vita effects. Developmental Psychology, 49(6), 1194-1218. https://doi.org/10.1037/ a0026913

Marsh, H. W., Morin, A. J., Parker, P. D., \& Kaur, G. (2014). Exploratory structural equation modeling: An integration of the best features of exploratory and confirmatory factor analysis. Annual Review of Clinical Psychology, 10, 85-110. https://doi.org/10.1 146/annurev-clinpsy-032813-153700

McDonald, R. P., \& Ho, M. -H. R. (2002). Principles and practice in reporting structural equation analyses. Psychological Methods, 7(1), 64-82. https://doi.org/10.10 37/1082-989X.7.1.64

Mena, F. J., Padilla, A. M., \& Maldonado, M. (1987). Acculturative stress and specific coping strategies among immigrant and later generation college students. Hispanic Journal of Behavioral Sciences, 9(2), 207-225. https://doi.org/10.1177/07399863 870092006

Morales, P. (2007). Estadística aplicada a las ciencias sociales. La fiabilidad de los test y escalas. Madrid: Universidad Pontificia Comillas. Recuperado de https://matcris5.fi les.wordpress.com/2014/04/fiabilidad-testsy-escalas-morales-2007.pdf

Muthén, L. K., \& Muthén, B. O. (2017). Mplus: statistical analysis with latent variables: User's Guide (Versión 8). Los Angeles: Autores.

Nunnally, J. C. (1987). Teoría psicométrica. México: Trillas.

Organización Internacional para las Migraciones. (2010). Informe sobre las migraciones en el mundo. Recuperado de http://publications.iom.int/system/files/p df/wmr_2010_spanish.pdf?language $=$ es

Organización Internacional para las Migraciones. (2018). Migración. 
Recuperado de https://www.un.org/es/secti ons/issues-depth/migration/

Padilla, A. M., Álvarez, M., \& Lindholm, K. J. (1986). Generational status and personality factors as predictors of stress in students. Hispanic Journal of Behavioral Sciences, 8(3), 275-288. https://doi.org/10.1177/07399863 860083006

Sternberg, R., \& Lee, K. (2013). Depressive symptoms of midlife Latinas: effect of immigration and sociodemographic factors. International Journal of Women's Health, 5, 301-308. https://doi.org/10.2147/IJWH.S4 3132

Suarez-Morales, L., Dillon, F. R., Szapocznik,, J. (2007). Validation of the Acculturative Stress Inventory for Children. Cultural Diversity and Ethnic Minority Psychology, 13(3), 216-224. https://doi.org/10.1037/10 99-9809.13.3.216

Fondo Internacional de Emergencia de las Naciones Unidas para la Infancia. International Displacement monitoring center. (2019). Protecting and supporting internally displaced children in urban settings. Recuperado de https://www.unicef.org/med ia/56191/file/Protecting\%20the\%20rights \%20of\%20internally\%20displaced\%20chil dren.pdf

Urzúa, A., Basabe, N., Pizarro, J. J., \& Ferrer, R. (2017). Afrontamiento del estrés por aculturación: inmigrantes latinos en Chile. Universitas Psychologica, 16(5), 1-13. https:/ /doi.org/10.11144/Javeriana.upsy16-5.aeai

Urzúa, A., Caqueo-Urízar, A., Calderón, C., \& Rojas, L. (2017). iEl apoyo social mediatiza el efecto negativo del estrés por aculturación en la salud? Un estudio en colombianos y peruanos inmigrantes en el norte de Chile. Interciencia, 42(12), 818-822. Recuperado de https://www.redal yc.org/articulo.oa?id $=33953770006$

Urzúa, A., Ferrer, R., Canales, V., Núñez, D., Ravanal, I., \& Tabilo, B. (2017). The influence of acculturation strategies in quality of life by immigrants in Northern Chile. Quality of Life Research, 26(3),
717-726. https://doi.org/10.1007/s11136-0 $16-1470-8$

Urzúa, A., Heredia, O., \& Caqueo-Urízar, A. (2016). Salud mental y estrés por aculturación en inmigrantes sudamericanos en el norte de Chile. Revista Médica de Chile, 144, 563-570. Recuperado de https://scielo .conicyt.cl/pdf/rmc/v144n5/art02.pdf

Notas

* Artículo de investigación. Esta investigación fue financiada por CONICYT PIA CIE160007. 